\title{
Comparison of Immature Platelet Fraction (IPF) in Patients with Central Thrombocytopenia and Peripheral Thrombocytopenia
}

\author{
Sobia Ashraf ${ }^{1}$, Sindhu Rehmann ${ }^{1}$, Zahid Asgher ${ }^{2}$, Ambareen Hamid ${ }^{1}$ and Samina Qamar ${ }^{1}$ \\ ${ }^{1}$ Department of Pathology, King Edward Medical University, Lahore, Pakistan \\ ${ }^{2}$ Department of Pathology Laboratory, Doctors Hospital, Lahore, Pakistan
}

\begin{abstract}
Objective: To determine immature platelet fraction (IPF) in patients with thrombocytopenia and correlation with their bone marrow findings and to determine its predictive value in differentiating peripheral (hyper destructive) thrombocytopenia from central (hypo-productive) thrombocytopenia.

Study Design: Cross-sectional comparative study.

Place and Duration of Study: King Edward Medical University (KEMU) and Doctors Hospital Lahore, from December 2018 to February 2019.

Methodology: Patients of both genders and all age groups with thrombocytopenia presenting for bone marrow biopsy were included in the study. Clinical data, complete blood count (CBC) sample in EDTA, bone marrow aspirate and trephine biopsy were obtained. Slides were prepared and reviewed. CBC sample was analysed for counts and IPF in Doctors Hospital Laboratory within 4 hours of collection.

Results: According to bone marrow findings, patients were grouped under two categories; Group 1 with central (hypo-productive group) thrombocytopenia and Group 2 with peripheral (hyper-destructive group). Groupl $(n=44)$ showed median interquartile range (IQR) IPF $8.2(4.6-16.7)$, which was significantly lower $(p<0.001)$ than that of Group $2(n=14)$ in which Median IQR IPF was 25.5 (15.2-39.3). A significant moderate degree negative (inverse) correlation was observed between platelet counts and IPF in thrombocytopenic patients in both groups by Pearson correlation. It was statistically significant at $p<0.001$ level. IPF in hyper-destructive group has $100 \%$ predictive value compared to $31 \%$ in hypo-productive group.

Conclusion: IPF is a useful parameter and can reliably identify patients having thrombocytopenia due to peripheral destruction. In other cases, IPF should be used in conjunction with other investigations like bone marrow biopsy etc.
\end{abstract}

Key Words: Immature platelet fraction, Complete blood count, Central thrombocytopenia, Peripheral thrombocytopenia, Bone marrow failure, Peripheral destruction.

How to cite this article: Ashraf S, Rehman S, Asgher Z, Hamid A, Qamar S. Comparison of Immature Platelet Fraction (IPF) in Patients with Central Thrombocytopenia and Peripheral Thrombocytopenia. J Coll Physicians Surg Pak 2020; 30(08):796-800.

\section{INTRODUCTION}

Complete blood count (CBC) provides an insight to hemopoietic activity. It gives details of blood cell counts (WBC count, red cell count, and platelet count), differential types of WBCs, RBC and platelet indices and hemoglobin values. Platelets are produced by megakaryocytes and released into the peripheral blood. Newly released platelets are larger in size and contain RNA content that can be stained by new methylene blue.

Correspondence to: Dr. Sobia Ashraf, Department of Pathology, King Edward Medical University, Lahore, Pakistan

E-mail: s.qayyum81@gmail.com

Received: November 05, 2019; Revised: July 31, 2020;

Accepted: August 13, 2020

DOI: https://doi.org/10.29271/jcpsp.2020.08.796
These platelets are termed as reticulated platelets. ${ }^{1}$ The immature platelet fraction (IPF) measured by Sysmex XN analyzer, is a variable that appraises young or immature platelets in peripheral blood as a measurable (proportional) value of the total platelet count (\%-IPF). They have a lifetime of $<24$ hours. The ideal reference range for IPF varies from $1.6-7.1 \%$ in adults, and $1.0-6.8 \%$ in children. $^{2-4}$

The average platelet count in human ranges between 150 and $450 \times 10^{9} / \mathrm{L}$. Thrombocytopenia (Platelet count less than $150 \times$ $10^{9} / \mathrm{L}$ in peripheral blood) may occur due to several causes, roughly divided into two main mechanisms: either an increased destruction, or a decreased production. It is crucial to distinguish between these two mechanisms for management. ${ }^{5}$ IPF can be considered as marker of thrombopoiesis as the percentage rises with adequate megakaryocytic activity and high platelet turnover; and decreases in low megakaryocytic activity and low platelet turnover. 
Table I: Clinical diagnosis of thrombocytopenia with summary of results of IPF $p=$ significant.

\begin{tabular}{|c|c|c|c|c|c|c|c|}
\hline Groups & Number & IPF \% Median (IQR) & p-value & $\begin{array}{l}\text { Platelet per } \\
\text { microliter } \\
\text { (mean) }\end{array}$ & $\begin{array}{c}\text { Mean age } \\
\text { years }\end{array}$ & Male & Female \\
\hline Group 1 (central thrombocytopenia) & 44 & $8.2(4.6-16.7)$ & \multirow{2}{*}{$<0.001$} & 80.8 & 37.9 & $\begin{array}{c}29 \\
(65.9 \%)\end{array}$ & $\begin{array}{c}15 \\
(34.1 \%)\end{array}$ \\
\hline Group 2 (peripheral thrombocytopenia) & 14 & $25.5(15.2-39.3)$ & & 43.6 & 44.8 & $\begin{array}{c}5 \\
(35.7 \%)\end{array}$ & $\begin{array}{c}9 \\
(64.3 \%)\end{array}$ \\
\hline
\end{tabular}

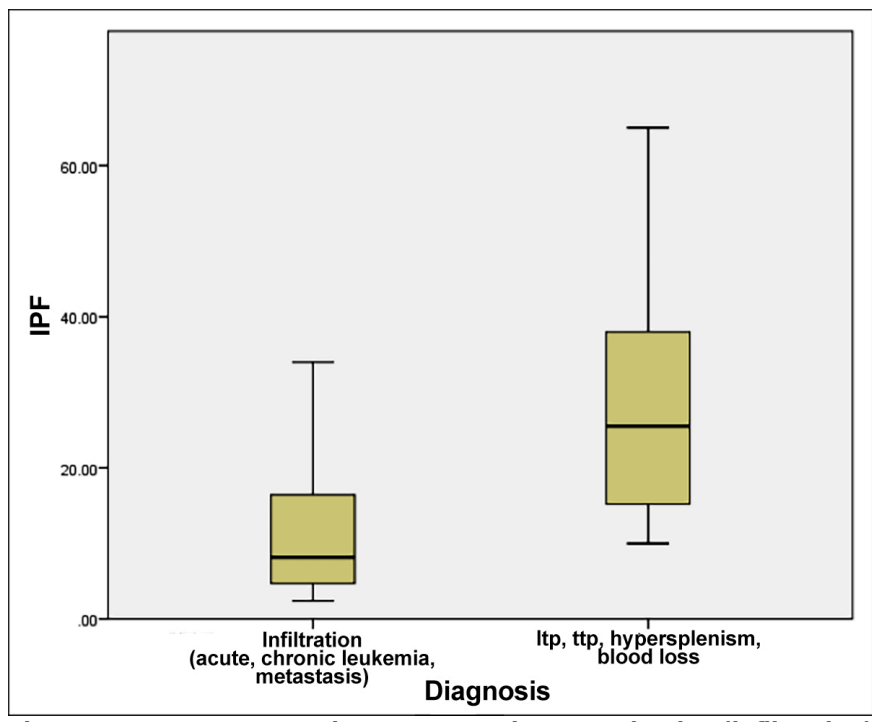

Figure 1: Mean IPF range in two groups hypo-productive (infiltration) = Group1; and hyper-destructive marrow (ITP, TTP, hypersplenism) = Group 2.

IPF is judged to be low/normal, if less than or equal to $7.7 \%$, and increased if $>7.7 \%$. Raised IPF levels are seen in conditions with high platelet turnover (hyper-destructive group) like disseminated intravascular coagulation (DIC), thrombotic thrombocytopenic purpura (TTP)/hemolytic uremic syndrome (HUS), immune thrombocytopenic purpura (ITP), and blood loss. Whereas, low levels such as $0.9-2.3 \%$ are observed in individuals with bone marrow suppression (hypo-productive group) such as aplastic anemia and other bone marrow failure syndromes, nutritional deficiencies and drug induced myelosuppression. ${ }^{2}$ It is found to be highly variable among patients with neoplastic marrow infiltration multiple myeloma, acute leukemia and medullary infiltration by solid tumours. ${ }^{6}$

Immature platelet fraction can also be an early predictor of bone marrow recovery after chemotherapy and stem cell transplantation. IPF values are also helpful in avoiding unnecessary platelet transfusions as the values increase several days prior to a rise in the platelet count. ${ }^{3}$ It has also been studied as prognostic marker in cardiovascular diseases. ${ }^{7}$ Diabetes mellitus ${ }^{8}$ and pregnancy-induced hypertension. ${ }^{9}$ IPF values are also found to be helpful in determining etiology of thrombocytopenia in liver cirrhosis patients. ${ }^{10}$

IPF is a relatively new marker in our setup and there is limited data available on the importance of IPF as a diagnostic marker in hypo productive thrombocytopenia.
The objective of this study was to determine IPF in patients presenting with thrombocytopenia and correlation of bone marrow findings in hematological disease and to determine predictive value of immature platelet fraction (IPF \%) in differentiating hyper-destructive/consumptive thrombocytopenia from hypo-productive thrombocytopenia.

\section{METHODOLOGY}

It was a cross-sectional comparative study conducted at King Edward Medical University (KEMU), Hospital Laboratory from December 2018 to February 2019. Inclusion criteria were patients of all ages and both genders presenting to the Hematology Department for bone marrow biopsy for hematological diseases, and with platelet counts less than $100 \mathrm{x}$ $10^{9} / \mathrm{L}$, confirmed after peripheral blood film review. Exclusion criteria were pseudo thrombocytopenia after peripheral blood film review and patients receiving prior treatment for their hematological disease.

After taking informed consent, a detailed personal and medical history was taken. Bone marrow aspirate and trephine biopsy were done from posterior iliac crest. Bone marrow aspirate slides were stained with Giemsa stain. The trephine biopsy was processed in standardised manner and stained with Hematoxylin and Eosin. Peripheral venous sample (taken as routine in bone marrow procedure) was drawn and stored in EDTA vial and was analysed for CBC on Sysmex kx 21 and IPF in Doctors Hospital Laboratory on Sysmex XN 550 within four hours of collection.

The data was statistically analysed by SPSS version 20 . The descriptive parameters were described in terms of frequencies and percentages; whereas, inferential parameters for Group 1, Group 2 were statistically analysed by median (IQR). Normality of data distribution is checked by ShapiroWilk test and was found to be non-parametric (asymmetrical, Figure 1); it was tested by Man-Whitney U-test at significance level $95 \%$ and $p$ value $<0.05$.

\section{RESULTS}

The study was carried out over a period of three months.A comparative cross-sectional study was carried out on 58 thrombocytopenic patients (platelet $<150 \times 10^{9} / \mathrm{L}$ ) based on clinical history and laboratory tests for bone marrow aspiration and biopsy. There were 34 (58.6\%) men and 24 (41.4\%) women (Table I). Age and gender for both Groups with IPF\% range in each Group was determined (Table I). Patients with the diagnosis of Iymphoma, leukemia, aplastic anemia, 
megaloblastic anemia and leishmaniasis were categorised as Group 1; whereas, patients with ITP, TTP, hypersplenism and DIC were placed in Group 2. There were 44 (75.9\%) patients in Group 1 (central thrombocytopenia) including 12(27.3\%) patients with leukemia, $17(38.6 \%)$ with infiltration by lymphoma, 8 (18.2\%) with aplastic anemia, 6 (13.6\%) with megaloblastic anemia, and $1(2.3 \%)$ had Leishmaniasis. There were 14 (24.1\%) patients in Group 2 (peripheral thrombocytopenia) and among them 5 (35.7\%) patients each had ITP and hypersplenism, 3 (21.4\%) had DIC and one $(7.1 \%)$ was suffering from TTP (Figure 1).

Using Mann-Whitney test, the Groupl ( $n=44)$ with hypo-productive thrombocytopenia showed median (IQR) IPF 8.2 (4.6-16.7), which was significantly lower $(p<0.001)$ than that of Group $2(n=14)$ hyper-productive thrombocytopenia in which median IPF (IQR) was 25.5 (15.2-39.3). A significant negative (inverse, -0.532) correlation was observed between platelet counts and IPF in thrombocytopenic patients in both Groups by Pearson correlation $(p<0.001)$. Predictive value was determined and found to be $100 \%$ in hyper-destructive group and was 31\% in hypo-productive group.

\section{DISCUSSION}

Platelets circulate in the peripheral blood and act as an integral element of homeostasis. The average platelet count in human beings ranges between 150 and $350 \times 10^{9} / \mathrm{L} .{ }^{1}$ Severe and very severe thrombocytopenia due to any cause have serious clinical implications and can be life-threatening. Immature platelet fraction is useful parameter for differentiating various causes of thrombocytopenia. Since IPF is a marker of platelet reactivity, it is also helpful in monitoring of numerous systemic diseases with impending danger of thrombosis. $^{7-10}$

The patients included in this study had thrombocytopenia and they presented for the workup. Diagnosis of etiology of thrombocytopenia was made on examination of bone marrow biopsy; and IPF levels were correlated with finding. In Group 1 (central thrombocytopenia) patients of leukemia, lymphoma, megaloblastic anemia, aplastic anemia and leishmaniasis were included; whereas, in Group 2 (peripheral thrombocytopenia), cases of ITP, TTP and hypersplenism were included. According to latest guidelines, bone marrow biopsy is not indicated for the diagnosis management of primary ITP; however, biopsy can be performed in certain situations and on physician's request. ${ }^{11}$

There were 44 patients in Group 1 and out of these, 29 (66\%) were males and $15(34 \%)$ were females. Fourteen patients in Group 2 included 5 (36\%) males and 9 (64\%) females. Median age in Groupl was 32.5 years and in Group 2 was 45 years. In similar study conducted by Arshi et al., males and females were 30 and 32 in ITP group; and 100 and 69 in non-ITP group. The median age for hypo-productive group was 32 years; and 23 years in hyper-destructive group. ${ }^{12}$
The platelets counts were analysed by CBC (complete blood counts) and confirmed on smear. Mean platelets counts in group1 ( $\mathrm{N}=44$ ) were $80.8 \times 10^{9} / \mathrm{L}$ and $43.6 \times 10^{9} / \mathrm{L}$ in group $2(\mathrm{~N}=14)$. In the study conducted by Arshi et al., the mean platelet count in hypo- productive group was 54 and in hyper-destructive group were 61. In that study, only CBC and IPF was done and cases of ITP were of primary type; whereas, in our study bone marrow was performed on these ITP patients which were either resistant to therapy or had secondary ITP; that is why mean platelet count was lower in this study. ${ }^{12}$

Mean platelet count was higher in Group 1 (hypo-productive group); whereas, was lower in Group 2 (hyper-destructive group). This is similar to the results of study by Lee et al. who also observed lower levels in the second group. ${ }^{13}$

IPF levels were measured and mean was calculated. Median (IQR) IPF of Group 1 showing decreased platelet production was 8.2 (4.6-16.7) and that of group 2 showing increased peripheral destruction were 25.5 (15.2-39.3). These results verify that IPF is markedly increased in peripheral destruction. Statistical analysis showed significantly lower values in hypo-productive group as compared to hyper-productive group; and negative correlation was also found between platelet count and IPF thrombocytopenia. This finding has been shown in different studies; and IPF has always been shown to be a reliable diagnostic marker of ITP. 3,13-15

Normal range of IPF is $1-7.7 \%$ and in Group 1, mean IPF was found to be above normal. Mean IPF in 12 patients of acute leukemia was $16.2 \%$, which was much higher than normal. This finding has also been observed by Strauß et al. who analysed IPF levels in children presented with thrombocytopenia; and IPF levels were raised in acute lymphoblastic leukemia. ${ }^{16}$ In the study by Psaila et al., platelet function were compared between AML/MDS and ITP patients. They found lower IPF levels in AML/MDS (11.2\%) patients than ITP patients (25.4\%). However, the mean IPF was higher than normal. These findings are exactly in accordance with this study. ${ }^{17}$

Mean IPF was calculated in 16 patients with Iymphoma. Out of these, 13 had bone marrow infiltration and 3 had no infiltration; but the marrow was hypo cellular, so the mean IPF was $6.5 \%$. In a study conducted by Vaughan et al., IPF was decreased in hypo- cellular patients and increased in malignant marrow infiltration. The discrepancy between the two values can be due to inclusion of hypo-cellular marrow patients in calculation of mean in this group in our study. ${ }^{18}$

In eight patients with aplastic anemia, mean IPF was found to be raised (14.7\%). This finding has also been seen by Abe et al. in their study, who observed normal levels in aplastic anemia. ${ }^{19}$ In study by Jung et al., IPF levels were lower $(3.5 \%)$ in aplastic anemia. However, the range was variable (range, 0.6-12.9\%). So the levels are variable in different patients of aplastic anemia and are perhaps to difference in stage of presentation. ${ }^{20}$ 
In the six patients of megaloblastic anemia, IPF value was $8.6 \%$ in this study. Monteagudo et al. included megaloblastic anemia patients in the group with central thrombocytopenia (decreased production) and the mean IPF and the range were 7.5 and $2-9.7 \%$, respectively. ${ }^{6}$ These findings are in accordance with the present study.

Total mean IPF in hypo-productive group in our study was $11.3 \%$. A study has been conducted by Cybulsaka et al. in 2017, and they compared efficacy of Sysmex analyser and CD Sapphire by Abbott in determining and comparing IPF levels in bone marrow failure and ITP patients. ${ }^{21}$ They also noted increased level (10.9\%) in bone marrow failure patients when samples run on $C D$ sapphire and observed inverse correlation between low platelet count and increased IPF due to thrombocytopenia of any cause. The reason behind this phenomenon as described by the author is accelerated release of immature platelets into the circulation which is driven by a state of low platelet count in blood similar to reticulocyte shift. They have suggested this as immature platelet (imPLT).

Comparative analysis between both thrombocytopenic groups showed statistically significant differences in IPF percentage $(p<0.001$, Table I). There was inverse correlation found between level of IPF\% and platelet count in all patients irrespective of diagnosis. The results of the studies by Arshi et al. and study by Lee et al. inverse correlation was found only in group with increased destruction. The reason of this discrepancy can be due to patient selection in hypo-productive group. We included the patients who presented for the workup and were not getting treatment; whereas, in study by Lee et al., patients receiving chemotherapy were included. ${ }^{12,13}$

Similarly, a recent study by Lee et al. showed platelet indices including IPF\% were measured in 31 patients with hyper-destructive/consumptive thrombocytopenia (14 with immune thrombocytopenic purpura and 17 with liver cirrhosis), and 34 patients with hypo- productive thrombocytopenia (4 with aplastic anemia and 30 with cancer who were undergoing chemotherapy). The platelet number in the hyper-destructive/consumptive thrombocytopenia group was significantly lower than that in the hypo-productive thrombocytopenia group $(p<0.001)$. The IPF\% was significantly higher in the hyper- destructive/consumptive thrombocytopenia group (median: $6.2 \%$ [IQR $4.3-10.3 \%$ ]) than the hypo-productive thrombocytopenia group (1.8\% [0.9-2.3\%]) (all p-values $<0.001)^{13}$

Arshi et al. also determined that there was significant inverse correlation of platelet count with IPF\%; the lower the platelet counts, the higher the IPF\%. The IPF\% value reflected the severity of platelet destruction. With a poor sensitivity of about $41.76 \%$ for non-ITP thrombocytopenia IPF\% may not be considered as a good biomarker for these cases. Further studies are indeed required to justify this conclusion. ${ }^{12,22}$

\section{CONCLUSION}

Immature platelet fraction is a useful parameter and it can reliably identify patients having thrombocytopenia due to peripheral destruction or utilisation. However, in cases with thrombocytopenia due to bone marrow diseases, IPF should be used in conjunction with other investigations like peripheral blood examination and bone marrow biopsy etc.

\section{ETHICAL APPROVAL:}

Ethical approval letters have been obtained from Departmental Board of Studies and Ethical Review Committee of King Edward Medical University and Ethical Review Committee of Doctors Hospital Lahore prior to initiation of the research work.

\section{PATIENTS' CONSENT:}

Patients were counselled and informed consents for research and publishing of data were taken prior to obtaining sample.

\section{CONFLICT OF INTEREST:}

There is no conflict of interest among the authors.

\section{AUTHORS' CONTRIBUTION:}

SA: Concept and design of study, analysis and draft formation.

SR: Concept and design of study, data collection and analysis.

ZA: Contribution to test performance, draft formation and review.

AH: Analysis, review and final approval of draft.

SQ: Data collection, analysis and results compilation.

\section{REFERENCES}

1. Liu QH, Song MY, Yang BX, Xia RX. Clinical significance of measuring reticulated platelets in infectious diseases. Medicine (Baltimore) 2017; 96(52):e9424. doi:10.1097/MD.0000000000009424

2. Shaikh MS, Ali SA. Clinical utility of immature platelet fraction - an advanced parameter in laboratory hematology. J Coll Physicians Surg Pak 2016; 26(9):798-9.

3. Imperiali CE, Arbiol-Roca A, Sanchez-Navarro L, Dastis-Arias M, Lopez-Delgado JC, Cortes-Bosch A, et al. Reference interval for immature platelet fraction on Sysmex XN haematology analyzer in adult population. Biochem Med (Zagreb) 28(1):010708. doi:10.11613/BM.2018.010708

4. Junxun Li, Ying Li, Juan O, Fan Z, Chujia L, Zhuangjian Ye, et al. Immature platelet fraction related parameters in the differential diagnosis of thrombocytopenia. Platelets 2020; 31(6):771-6. doi: 10.1080/09537104.2019.1678118.

5. Negash M, Tsegaye A, G/Medhin A. Diagnostic predictive value of platelet indices for discriminating hypo productive versus immune thrombocytopenia purpura in patients attending a tertiary care teaching hospital in Addis Ababa, Ethiopia. BMC Hematol 2016; 16:18. doi:10.1186/ s12878-016-0057-5.

6. Monteagudo M, Amengual J, Munoz L, Soler J, Roig I, Tolosa C. Reticulated platelates as a screening test to identify 
thrombocytopenia aetiology. QJM 2008; 101(7):549-55. doi:10.1093/qjmed/hcn047.

7. Anetsberger A, Blobner M, Haller B, Schmid S, Umgelter K, Hager $T$, et al. Immature platelets as a novel biomarker for adverse cardiovascular events in patients after non-cardiac surgery. Thromb Haemost 2017; 117(10):1887-95. doi:10.1160/TH16-10-0804

8. Neergaard PS, Hvas AM, Grove EL, Larsen SB, Gregersen S, Kristensen SD. The influence of haemoglobinalc levels on platelet aggregation and platelet turnover in patients with coronary artery disease treated with aspirin. PLoS One 2015; 10(7):e0132629. http://doi.org/10.1371/journal. pone.0132629.

9. Moraes D, Munhoz TP, Pinheiro da Costa BE, Hentschke MR, Sontag F, Silveira Lucas L, et al. Immature platelet fraction in hypertensive pregnancy. Platelets 2016; 27(4):333-7. doi: 10.3109/09537104.2015.1101060.

10. Rauber P, Lammert F, GrotemeyerK, Appenrodt B. Immature platelet fraction and thrombopoietin in patients with liver cirrhosis: A cohort study. PLoS One 2018; 13(2): e0192271. http://doi.org/10.1371/journal.pone.0192271

11. Matzdorff A, Meyer O, Ostermann H, Kiefel V, Eberl W, Kühne T, et al. Immune Thrombocytopenia - current diagnostics and therapy: Recommendations of a Joint working group of DGHO, ÖGHO, SGH, GPOH, and DGTI. Oncol Res Treat 2018; 41(suppl 5):1-30. doi: 10.1159/000492187.

12. Naz A, Mukry SN, Shaikh MR, Bukhari AR, Shamsi TS. Importance of immature platelet fraction as predictor of immune thrombocytopenic purpura. Pak J Med Sci 2016; 32(3): 575-79. doi:10.12669/pjms.323.9456.

13. Lee $Y K$, Jeon K, Kim M, Lee E, Lee J, Lee JS, et al. Immature platelet fraction: A useful marker for identifying the cause of thrombocytopenia and predicting platelet recovery. Blood 2018; 132 (Supplement 1):128. doi.org/10.1182/blood-2018-99113202.

14. Ferreira FLB, Colella MP, Medina SS, Costa-Lima C, Fiusa MML, Costa LNG, et al. Evaluation of the immature platelet fraction contribute to the differential diagnosis of heredi-tary, immune and other acquired thrombocytopenias. Sci
Rep 2017; 7(1): 3355. doi:10.1038/s41598-017-03668-y.

15. Cho YG, Lee JH, Kim DS, Lee HS, Choi SI. Clinical usefulness of the simple technique to diagnose thrombocytopenia using immature platelet fraction. Korean J Lab Med 2007; 27(1):1-6. doi:10.3343/kjlm.2007.27.1.1.

16. Strauß G, Vollert C, Weimann A, Stackelberg AV, Weimnn A, Gaedicke G, et al. Immature platelet count: A simple parameter for distinguishing thrombocytopenia in pediatric acute lymphocytic leukemia immune thrombocytopenia. Pediatr Blood Cancer 2011; 57(4):641-7. doi:10.1002/ pbc.22907.

17. Psaila B, Bussel JB, Frelinger AL, Babula B, Linden MD, Li Y, et al. Differences in platelet function in patients with acute myeloid leukemia and myelodysplasia compared to equally thrombocytopenic patients with immune thrombo-cytopenia. J Thromb Haemost 2011; 9(11):2302-10. doi: 10.1111/j.1538-7836.2011.04506.

18. Vaughan J, Wiggill T, Munster M. Immature platelet fraction levels in a variety of bone marrow pathologies in South African HIV-positive patients with thrombocytopenia. Hematology 2014; 19(7):417-23. doi: 10.1179/16078 45413Y 0000000143.

19. Abe $Y$, Wada H, Tomatsu H, Sakaguchi A. Nishioka J, Yabu Y, et al. A simple technique to determine thrombopoiesis level using immature platelet fraction (IPF). Thromb Res 2006; 118(4):463-9. doi: 10.1016/j.thromres.2005.09.007.

20. Jung $\mathrm{H}$, Jeon $\mathrm{HK}, \mathrm{Kim} \mathrm{HJ}$, Kim SH. Immature platelet fraction: Establishment of a reference interval and diagnostic measure for thrombocytopenia. Korean J Lab Med 2010; 30(5):451-9. doi:10.3343/kjlm.2010.30.5.451.

21. Cybulska A, Meintker L, Ringwald J, Krause SW. Measurements of immature platelets with haematology analysers are of limited value to separate immune thrombocytopenia from bone marrow failure. Br J Haematol 2017; 177(4): 612-9. doi:10.111 1/bjh.14628

22. Jeon M, Choi H, Yu SE, Kang KW, Lee BH, Kim DS, et al. Immature platelet fraction based diagnostic predictive model for immune thrombocytopenic purpura. Blood 2018; 132(Supplement 1):1149. doi.org/10.1182/blood-2018-99117754. 